Journal of Adhesion Science and Technology 25 (2011) 27-40

\title{
Prevention of Ice Accretion on Aluminum Surfaces by Enhancing Their Hydrophobic Properties
}

\author{
N. Saleema a,*, M. Farzaneh a, R. W. Paynter b and D. K. Sarkar a,** \\ a Atmospheric Icing Engineering of Power Networks (CIGELE) and Atmospheric Icing of \\ Power Network Equipment (INGIVRE), Université du Québec à Chicoutimi, 555 Blvd. \\ Université, Chicoutimi, Québec, G7H 2B1, Canada \\ b INRS Énergie Matériaux Télécommunications, 1650 Boulevard Lionel-Boulet, \\ Varennes, Québec, J3X 1S2, Canada
}

\section{DOI:10.1163/016942410X508064}

\begin{abstract}
The accretion of ice on the surfaces of power network systems, aircraft, communication networks, etc., is known to cause serious problems that often lead to costly safety issues. An ideal solution would be to prevent ice from accumulating in the first place, rather than waiting for ice to accrete and then to de-ice which is both time-consuming and expensive. This may be accomplished by depositing coating materials that are icephobic.

A low dielectric constant surface is expected to reduce the adhesion of ice due to the screening of mirror charges, thereby eliminating one of the strongest interaction forcesthe electrostatic force of attraction-at the ice-surface interface. Superhydrophobic surfaces, which demonstrate high water-repellency due to the negligible contact area of water with these surfaces, are also expected to minimize the contact area of ice. In the present research work, both concepts were studied by producing superhydrophobic nanorough low- $\varepsilon$ (dielectric) surfaces on aluminum. Superhydrophobic properties were achieved on surfaces of aluminum by creating a certain nanoroughness using a chemical etch followed by 'passivation' of the surface by a low surface energy coating of rf-sputtered Teflon, providing a water contact angle greater than $160 \circ$. The same behavior is reported even when the nanorough substrates were coated with dielectric thin films of $\mathrm{ZnO}$ (lower $\varepsilon$ ) or TiO2 (higher $\varepsilon$ ) prior to passivation. It is found that the superhydrophobic nanorough low energy surfaces are also icephobic and the presence of a low dielectric constant surface coating of Teflon $(\varepsilon=2)$ allows a considerable reduction of the ice adhesion strength. Ice adhesion strengths were determined using a centrifugal ice adhesion test apparatus.
\end{abstract}

\section{Keywords}

Superhydrophobicity, icephobicity, aluminum surfaces, low dielectric coating, chemical etching, sol-gel, rfs, puttered Teflon, scanning electron microscopy, X-ray photoelectron spectroscopy

\section{Introduction}

Icing on various structures is a serious and significant problem posing safety issues and system operation problems. Examples of the most important structures exposed to ice and causing a threat to safety are hydroelectric power lines, hightension insulators, wind turbines, aircraft, the space shuttle, ship hulls, off-shore ocean structures, highways, runways, automobile wind-shields, and domestic structures such as roofs and windows. 
It is well known that ice bonds strongly to almost all surfaces making its removal difficult and creating the need for a technological solution to aid in the debonding of ice.Current strategies to eliminate or reduce ice buildup such as chemical, mechanical or thermal deicing methods have several disadvantages. The need to apply and maintain a sufficient quantity of deicers, such as freezing point depressants employed on highways and deicing fluids such as ethylene glycol and propylene glycol used on aircraft wings, makes it both time-consuming and expensive to continually contend with ice.

Furthermore, the deicing fluids are toxic and environmentally unsafe. The mechanical removal of ice from surfaces by scraping or vibrating the system vigorously [1] can cause damage to the surfaces, reducing the service life of the system. Heating the surface to melt the ice can be an effective method, but it requires a large supply of energy [2]. However, none of these techniques prevents ice from forming or accumulating, but these techniques are used only after there is an ice buildup or accumulation. Therefore, making a surface to which ice would not stick at all would be an ideal and economical solution to eliminate the use of complex de-icing techniques. Such a surface to which ice would not adhere would be termed an 'icephobic' surface. The strong adhesion of ice to materials is due mainly to the properties of the ice-solid interface where polar water molecules strongly interact with the solid.

The processes involved in ice adhesion can be attributed to three different kinds of interactions: chemical bonding, Lifshitz-van derWaals interactions, and electrostatic interactions [3]. Ideally, all these three types of interactions must be eliminated to have zero or much reduced ice adhesion. It is the electrostatic forces between charges at the ice surface and mirror charges induced in the solid substrate that have been found to largely dominate the adhesion of ice to surfaces. The use of materials with a very low dielectric constant would reduce the electrostatic interactions at the ice-dielectric interface and result in significant reduction in the adhesion strength of ice.

Wettability is another important property which can significantly influence the adhesion of ice to surface structures. Wettability or the wetting behavior is in practice often evaluated by measuring the contact angle of a water droplet on the surface. A surface with contact angle (CA) less than 90 。 is said to be hydrophilic and a surface with a CA greater than 90 ० is said to be hydrophobic, a definition commonly encountered in the literature [4]. A surface with a CA greater than 150', however, is termed 'superhydrophobic', and on such a surface, water droplets would roll off with nearly zero wetting, a phenomenon seen on the surfaces of many leaves and insect body parts, evolved mainly for the purpose of protection [5-7]. One commonly known example is the lotus leaf surface, for which the phenomenon is also termed the 'lotus effect' [7]. Such a phenomenon on these surfaces results from a combination of a rough surface topography and a low surface energy coating present on these surfaces. Nature teaches the human kind many great things in science and provides solutions to many engineering problems as well. Engineering superhydrophobic surfaces is one among its great teachings where scientists and engineers have learnt that geometry (surface roughness) and chemistry (low surface energy) must co-exist in order to achieve the water repellent properties as observed on the self-clean lotus leaves [7] and many insects and animal bodies [5, 6]. We have previously engineered such surfaces employing various methods such as chemical bath deposition [8], galvanic exchange reactions [9], chemical etching [10] followed by passivating with low surface energy organic molecules or by coating with ultrathin films of low surface energy rf-sputtered Teflon. Recently Sarkar et al. [11] have also produced superhydrophobic silver surfaces in one-step where they demonstrated creation of roughness and lowering of surface energy in a single step during silver deposition. The importance of nanoroughness and chemical composition on the superhydrophobic properties have also been emphasized by Rios et al. both 
experimentally and theoretically $[12,13]$. A recent book entitled 'Superhydrophobic Surfaces' edited by A. Carré and K. L. Mittal discusses the concept and ways to fabricate superhydrophobic surfaces illustrating several applications of these surfaces [14]. All these investigations emphasize the importance of the co-existence of roughness and low surface energy in obtaining superhydrophobic properties on a surface, i.e., the water droplets roll off these surfaces without wetting them.

The interactions of both water and ice with a surface should be similar; therefore, the adhesion strength of ice would be expected to be lower against surfaces which water would not wet. In other words, if a surface is engineered in such a way that a water droplet does not wet it (i.e., it is a superhydrophobic surface), ice also would not be expected to adhere to it.

In the present paper, we report that etched aluminum surfaces rendered superhydrophobic by the application of a Teflon® coating by rf-sputtering show icephobicity with zero ice adhesion under freezing conditions. The effect of the dielectric constant was also investigated by coating the nanostructured aluminum surfaces with materials of different dielectric constants. The presence of rough nanostructures created on the aluminum surfaces was confirmed via scanning electron microscopy. X-ray photoelectron spectroscopy confirmed the presence of various elements of interest following coating with the different dielectrics.

\section{Experimental}

2.1. Creation of Rough Nanostructures on Aluminum Surfaces Rolled sheets of an AA6061 aluminum alloy (Al 97.9 wt\%, Mg 1.0 wt\%, Si 0.60 wt\%, Cu 0.28 wt\%, Cr 0.20 wt\%) of dimensions $32 \mathrm{~mm} \times 50 \mathrm{~mm}$ and thickness $1.05 \mathrm{~mm}$ used as substrates were ultrasonically cleaned for 20 min with 1\% LIQUINOX (Sigma Aldrich) followed by ultrasonication in de-ionized water for $20 \mathrm{~min}$. Rough nanostructures were created on clean aluminum surfaces by chemically etching using 40 vol\% aqueous hydrochloric acid $(\mathrm{HCl})$ for about three minutes. The etched coupons were then rinsed in running tap water to stop further reaction and cleaned by ultrasonication in de-ionized water to remove any debris followed by drying in a clean and closed oven for several hours to remove excess water prior to further coatings.

2.2. Dielectric Coatings on Rough Nanostructured Aluminum Surfaces Three dielectrics-TiO2, ZnO and Teflon® (rf-sputered)-were coated on the flat as well as nanorough nanostructured aluminum surfaces. The dielectric coatings of $\mathrm{TiO} 2$ and $\mathrm{ZnO}$ were also coated with rf-sputtered Teflon ${ }^{\circledR}$ to obtain superhydrophobicity on these surfaces. $\mathrm{TiO} 2$ and $\mathrm{ZnO}$ were coated via a sol-gel spin-coating technique. The sol-gel procedures described in the literature were followed for coating the two dielectrics whose dielectric constants are in the lower range of $\sim 8$ for $\mathrm{ZnO}$ as reported by Alexander et al. [15] and in the higher range of $\sim 80$ for TiO2 as reported by Sarkar and coworkers [16, 17]. The physical properties (not detailed in this paper) such as the crystal structure and thickness of the dielectric coatings have been investigated by X-ray diffraction (XRD) and Rutherford backscattering spectrometry (RBS) measurements, respectively and were found to be consistent with literature values. It is, therefore, reasonable to expect that the electrical properties will also be similar to those described by Alexander et al. [15] and Sarkar and coworkers [16, 17], although the dielectric constant measurements were notperformed.

For the coating of $\mathrm{TiO} 2$, a stock solution of $0.4 \mathrm{M} \mathrm{TiO} 2$ sol was prepared by diluting the tetrabutoxy titanium (TBOT) (C16H36O4Ti) precursor (Sigma Aldrich) with ethanol at a $1: 1$ volume ratio under constant stirring at $500 \mathrm{rpm}$ for $10 \mathrm{~min}$ at room temperature. $A$ desired amount of the stock $\mathrm{TiO} 2$ sol that had been aged for $48 \mathrm{~h}$ was deposited dropwise onto the aluminum substrate, spread using a spincoater (Laurell Tech, model 
WS-400B-6NPP) at $4000 \mathrm{rpm}$ for $30 \mathrm{~s}$, dried on a hotplate at $120 \circ \mathrm{C}$ for $30 \mathrm{~min}$, and annealed in air at $450 \circ \mathrm{C}$ for $30 \mathrm{~min}$.

For the coating of $\mathrm{ZnO}$, a stock solution of $0.5 \mathrm{M} \mathrm{ZnO}$ sol was prepared by dissolving $10.975 \mathrm{~g}$ of zinc acetate dihydrate $((\mathrm{CH} 3 \mathrm{CO} 2) 2 \mathrm{Zn} \cdot 2 \mathrm{H} 2 \mathrm{O})$ (Sigma Aldrich) in $100 \mathrm{ml}$ of methanol under constant stirring at $500 \mathrm{rpm}$ for $30 \mathrm{~min}$ on a hotplate maintained at $80{ }^{\circ} \mathrm{C}$. A desired amount of the stock $\mathrm{ZnO}$ sol that had been aged for $48 \mathrm{~h}$ was deposited dropwise onto the aluminum substrates and spin coated at $3000 \mathrm{rpm}$ for $30 \mathrm{~s}$, dried on a hotplate at $120{ }^{\circ} \mathrm{C}$ for $30 \mathrm{~min}$, and annealed in air at $450{ }^{\circ} \mathrm{C}$ for $30 \mathrm{~min}$.

Teflon® was sputtered onto the sol-gel spin-coated oxide surfaces using an Ar plasma in an inductively coupled plasma reactor (Plasmionique Inc., Varennes, Canada) by applying a power of $50 \mathrm{~W}$. The distance between the Teflon® target and the substrate was $30 \mathrm{~cm}$. The sputtering process was carried out for $\sim 20$ min at an Ar pressure of 20 $\mathrm{mTorr}$ in the chamber during process. The base pressure was $2 \times 10-6$ Torr.

\subsection{Characterization}

The presence of a rough nanostructure on the aluminum surfaces following chemical etching was confirmed by examination of images obtained using field emission scanning electron microscopy (FEGSEM, LEO 1525). X-ray photoelectron spectroscopy (XPS) (ESCALAB 220iXL, INRS-ÉMT, Varennes, Canada) was used for the surface chemical analyses of the dielectric coatings prior to and after ice adhesion tests. The wetting properties of the surfaces coated with the three dielectrics were investigated using a contact angle goniometer (Krüss $\mathrm{GmbH}$, Germany).

A water drop of volume $\sim 5 \mu \mathrm{l}$ was suspended from the syringe needle and brought into contact with the superhydrophobic surface using a computer controlled device provided by Krüss $\mathrm{GmbH}$. The advancing and receding contact angles were measured by holding the needle stationary and moving the sample in one direction. The static contact angle data were acquired by fitting the symmetric water drops using the Laplace-Young equation and the advancing and receding contact angles were determined from the asymmetric water drops using the tangent-2 method [18]. Icephobicity tests were performed by exposing the sample surfaces to a freezing drizzle of supercooled water droplets at $-10^{\circ} \mathrm{C}$ in a wind tunnel at a fixed wind speed of $10 \mathrm{~m} / \mathrm{s}$ in order to simulate atmospheric icing conditions in natural outdoor situations.

The samples mounted on untreated supports were placed vertically in the wind tunnel perpendicular to the direction of flow of the freezing drizzle. Following the accumulation of ice on the sample surfaces, the adhesion strength of ice was determined using a centrifugal ice adhesion test apparatus (CAT), which was designed and developed inhouse at CIGELE laboratories following the intensive use by Laforte and Beisswenger [19] as a standard method for testing various surfaces for ice adhesion strength. In this method, an aluminum beam (32 $\mathrm{mm}$ wide and $30 \mathrm{~cm}$ long) with the test sample $(32 \mathrm{~mm}$ $\times 50 \mathrm{~mm}$ ) and accumulated ice attached to one end, and a counter weight attached to the other end to balance the beam, is fixed in the centrifuge test chamber maintained at $-10 \cdot \mathrm{C}$. The beam is then rotated at increasing speeds resulting in a controlled ramp of the centrifugal force. When this force reaches the adhesion force of ice, the ice detaches from the sample surface.

The exact rotation speed at the time of ice detachment is determined from the computer software developed in-house. The adhesion force $F$ is then determined using the formula $F=m r \omega 2$, where $m$ is the mass of ice; $r$ is the radius of the beam; and $\omega$ is the rotation speed. The shear adhesion strength $(\tau=F / A)$ of ice is then determined from the apparent area $A$ of the sample surface which was in contact with ice.

\section{Results and Discussion}


The chemical reaction of $\mathrm{HCl}$ with aluminum removes some material from the aluminum surface leading to the creation of a certain roughness in nanometric scale on its surface. Figure 1 shows the FESEM image of an aluminum surface etched in $40 \mathrm{vol} \% \mathrm{HCl}$ and the inset of Fig. 1 shows the aluminum surface prior to $\mathrm{HCl}$ etching.

The formation of a rough coral-like nanostructure following etching is evident from the FESEM image, and contributes in combination with a modified surface chemistry to obtaining superhydrophobicity [8-13, 20]. The morphological features are found to be retained following deposition of the different dielectric materials on the etched aluminum surfaces as confirmed by FESEM.

The presence of $\mathrm{TiO} 2, \mathrm{ZnO}$ and rf-sputtered Teflon on the nanopatterned Al surfaces was confirmed from the XPS spectra. Figures 2 and 3 show the survey spectra of TiO2 and $\mathrm{ZnO}$ sol-gel coatings, respectively. The survey spectrum of TiO2 (Fig. 2) shows strong signals of Ti2p and O1s as well as strong Ti LMM Auger peak with Ti2p3/2 and Ti2p1/2 peaks at $458.85 \mathrm{eV}$ and $464.53 \mathrm{eV}$, respectively and the $01 \mathrm{~s}$ corresponding to $\mathrm{TiO} 2$ at $530.1 \mathrm{eV}$.

Similarly, the survey spectrum of $\mathrm{ZnO}$ in (Fig. 3) shows strong signals of $\mathrm{Zn} 2 \mathrm{p}$ and $\mathrm{O} 1 \mathrm{~s}$ as well as a strong Zn LMM Auger peak with the Zn2p3/2 peak at $1021.6 \mathrm{eV}$ and the $\mathrm{O} 1 \mathrm{~s}$ peak of $\mathrm{ZnO}$ at $530.28 \mathrm{eV}$.

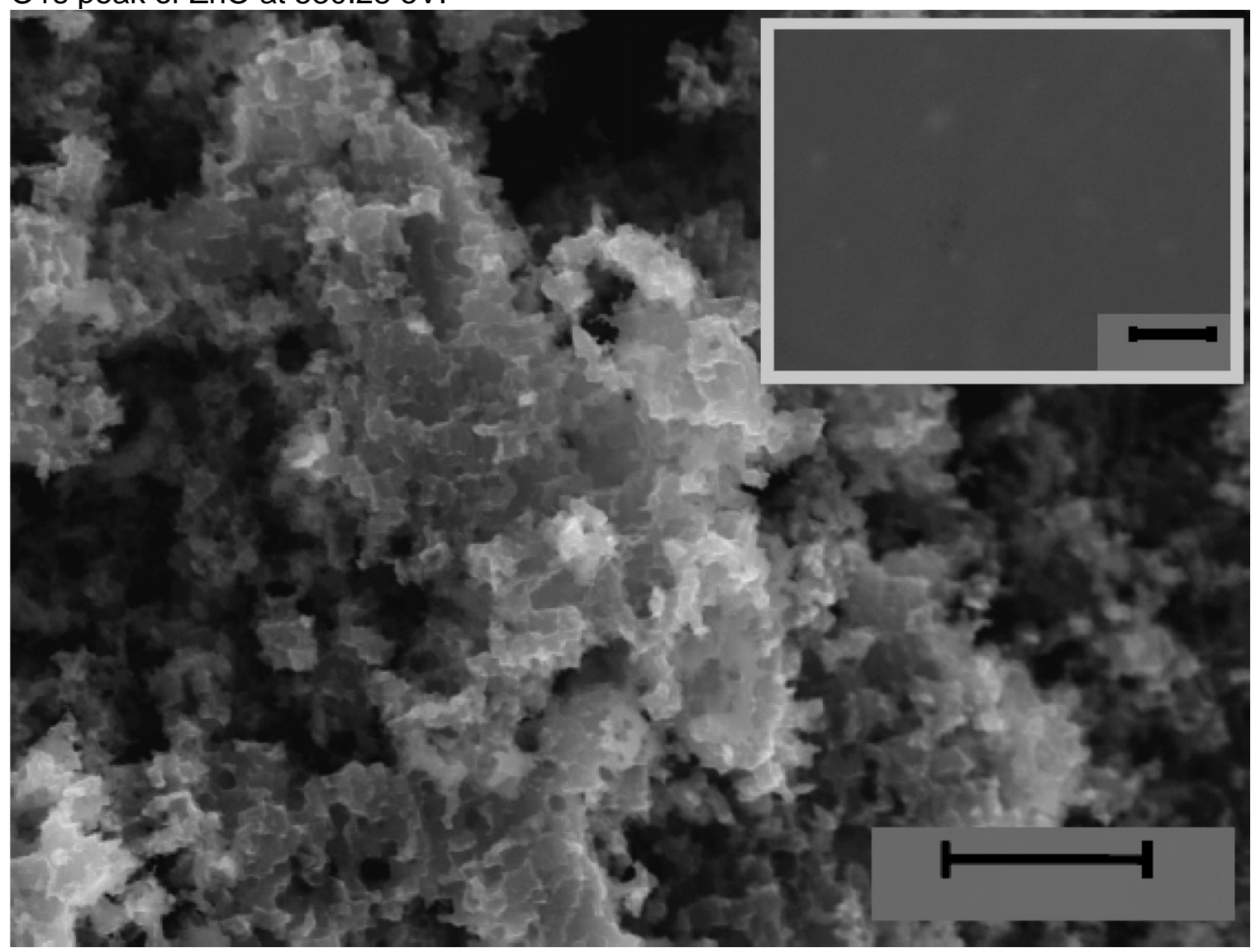

Figure 1. FESEM image of $\mathrm{HCl}$-etched aluminum surface; inset shows the aluminum surface before etching.

The survey spectrum of rf-sputtered Teflon, shown in Fig. 4, show strong peaks of $C$ and $\mathrm{F}$ confirming the presence of a coating of Teflon®. Figure 5 shows the $\mathrm{C} 1 \mathrm{~s}$ core level spectrum of rf-sputtered Teflon resolved into seven components: -CF3 (293.50 eV), - 
CF2 (291.43 eV), -CF-CFn (289.35 eV), -C-F (287.84 eV), -C-CFn (286.92 eV), -C-C $(285 \mathrm{eV})$ and $-\mathrm{C}-$ Metals $(283.20 \mathrm{eV})$. The relative proportions of all seven components resolved from the $\mathrm{C} 1 \mathrm{~s}$ peak fit are summarized in Table 1. The binding energy of $\mathrm{F} 1 \mathrm{~s}$ from rf-sputtered Teflon as shown in the inset of Fig. 5 was $688.5 \mathrm{eV}$. Wetting properties were investigated using CA measurements. Water droplets were found to spread completely on the rough coral-like nanostructure of the etched

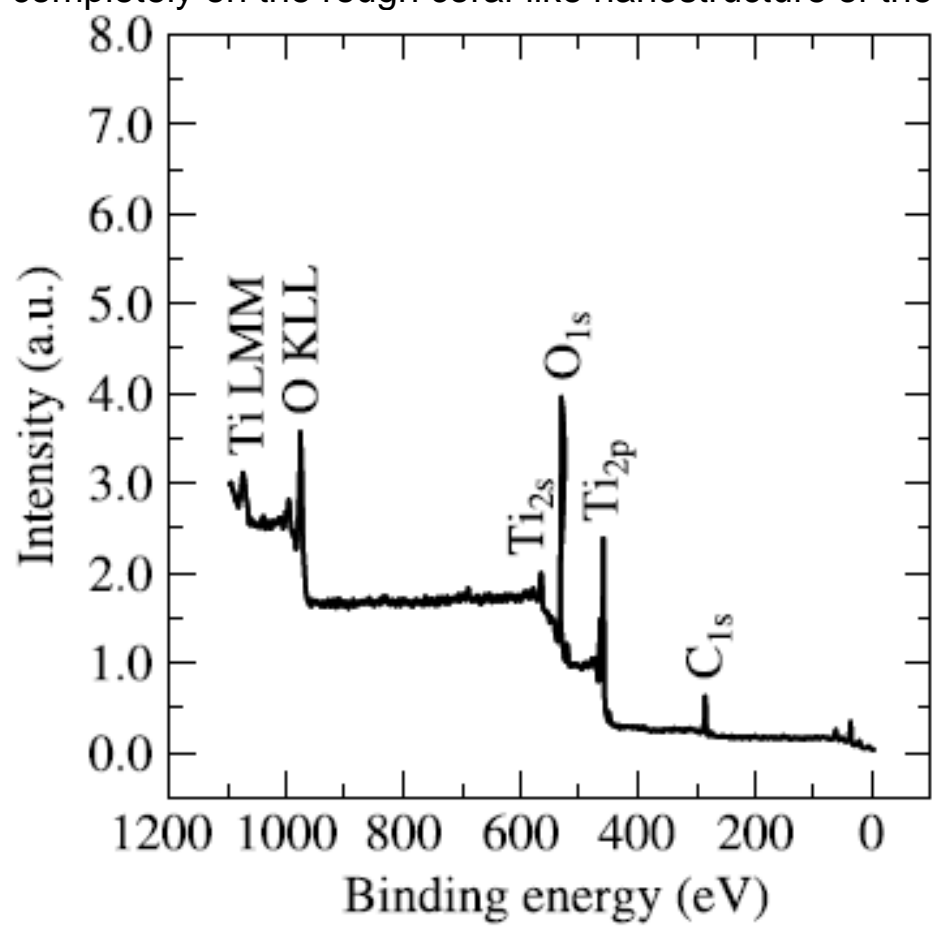

Figure 2. XPS survey spectrum of sol-gel TiO2 coating.

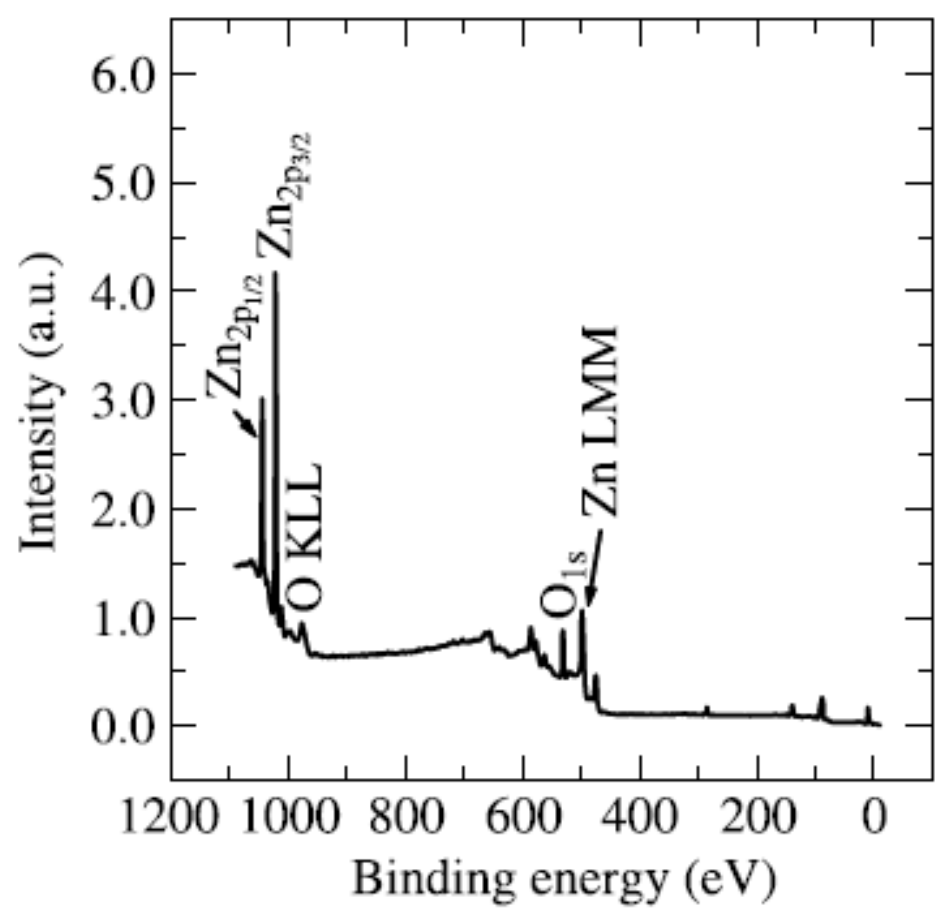


Figure 3. XPS survey spectrum of sol-gel $\mathrm{ZnO}$ coating.

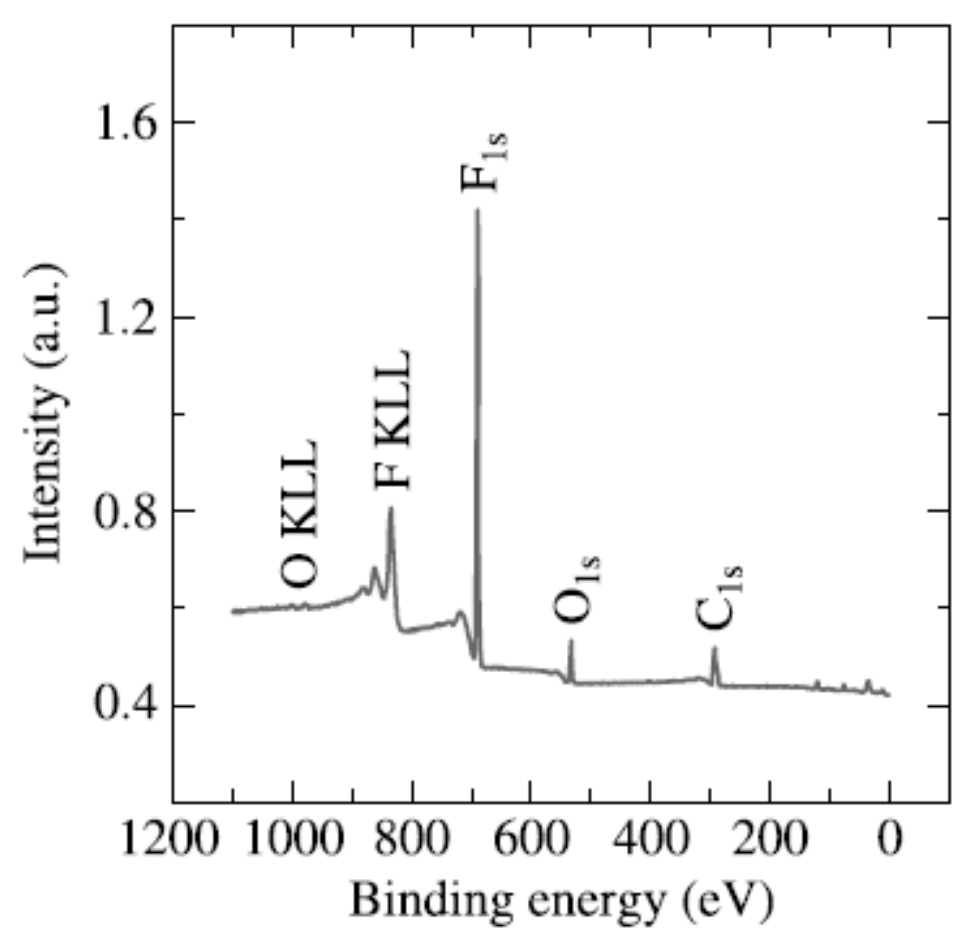

Figure 4. Survey spectrum of rf-sputtered Teflon coating. 


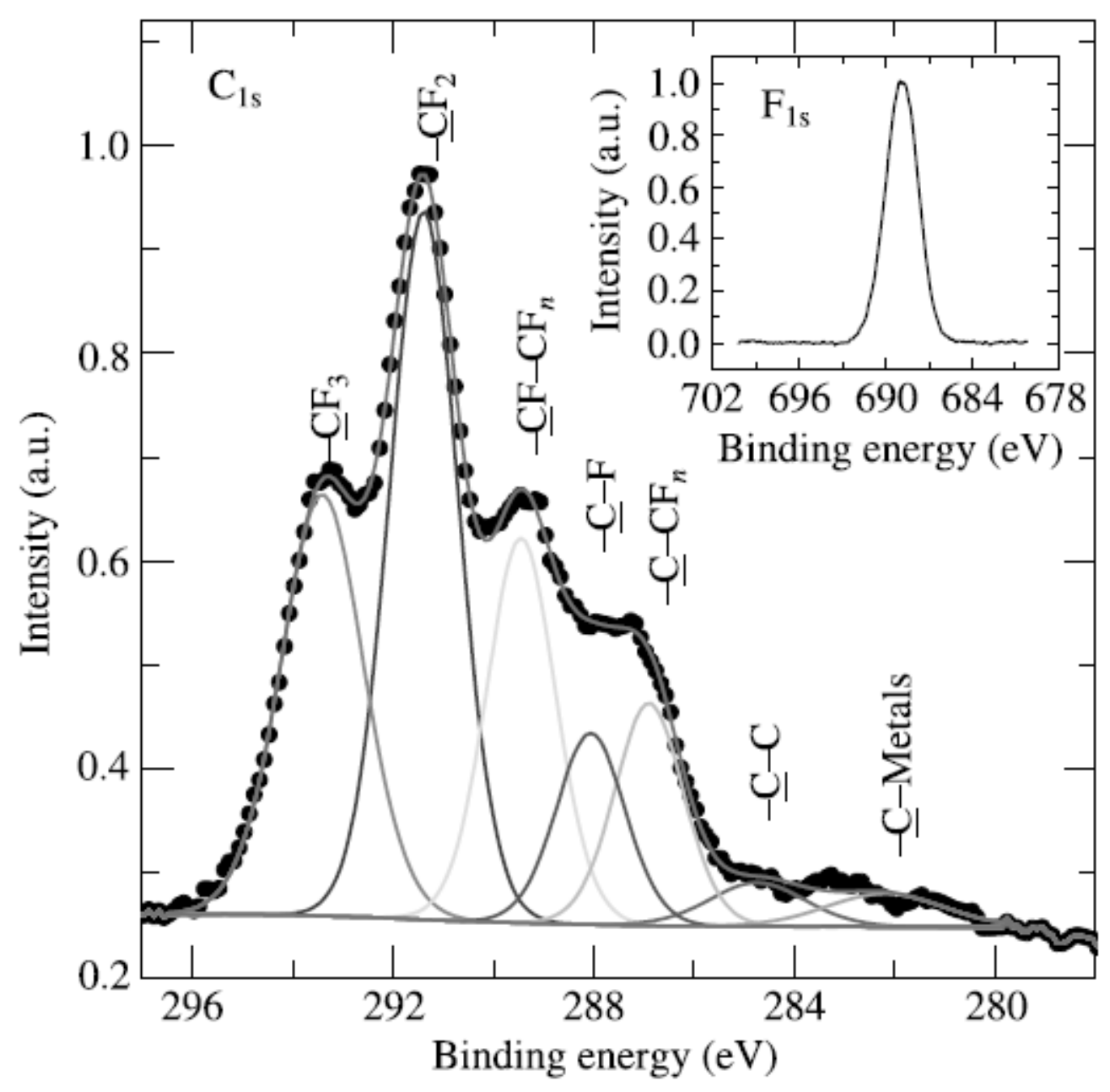

Figure 5. XPS high-resolution spectra of C1s and F1s, (inset) spectrum of an rfsputtered Teflon coating.

aluminum surfaces demonstrating hydrophilicity. A similar behavior was observed on ZnO-coated etched Al (ZCEA) surfaces. A small CA of $\sim 25^{\circ}$ was measured on the TiO2coated etched Al (TCEA) surfaces, still showing hydrophilic behavior. The reason for TCEA providing a certain value for contact angle could be 
Table 1.

Summary of $\mathrm{C}_{1 \mathrm{~s}}$ peak fit of rf-sputtered Teflon

\begin{tabular}{llc}
\hline Component & $\mathrm{BE}(\mathrm{eV})$ & Atom $(\%)$ \\
\hline $\mathrm{C}_{1 \mathrm{~s}}\left(-{ }_{\mathrm{C}} \mathrm{CF}_{3}\right)$ & 293.50 & 22.3 \\
$\mathrm{C}_{1 \mathrm{~s}}\left(-\underline{\mathrm{C}} \mathrm{F}_{2}\right)$ & 291.43 & 37.7 \\
$\mathrm{C}_{1 \mathrm{~s}}\left(-\underline{\mathrm{C}}-\mathrm{CF}_{n}\right)$ & 289.35 & 19.8 \\
$\mathrm{C}_{1 \mathrm{~s}}(-\underline{\mathrm{C}}-\mathrm{F})$ & 287.84 & 7.6 \\
$\mathrm{C}_{1 \mathrm{~s}}\left(-\underline{\mathrm{C}}-\mathrm{CF}_{n}\right)$ & 286.92 & 10.0 \\
$\mathrm{C}_{1 \mathrm{~s}}(-\underline{\mathrm{C}}-\mathrm{C})$ & 285 & 1.1 \\
$\mathrm{C}_{1 \mathrm{~s}}(-\underline{\mathrm{C}}-$ Metals $)$ & 283.20 & 1.4 \\
\hline
\end{tabular}

presence of some contamination on TCEA surface or the difference in dielectric constant as compared to $\mathrm{ZnO}$. However, with contact angle value well below $90^{\circ}$, all these surfaces fall into the hydrophilic regime. All these surfaces presenting certain nanoroughness exhibited superhydrophobicity when coated with low surface energy rfsputtered Teflon providing a $C A>160^{\circ}$ and contact angle hystereses $(C A H)<5^{\circ}$. Due to the very low $\mathrm{CAH}$, which is the difference between the advancing and receding contact angles measured on the two sides of a droplet advancing across the surface, the water droplets were found to roll off the superhydrophobic etched aluminum surfaces even at a slightest tilt of the sample. Such low hysteresis values are comparable with the very low hysteresis of $\sim 1.4 \circ$ reported on stearic acid passivated $\mathrm{ZnO}$ nanotowers [8] and $<5^{\circ}$ reported on stearic acid modified silver nanoparticles on copper substrates [9]. A flat aluminum surface (CA of $\sim 74 \circ$ ) when coated with rf-sputtered Teflon provided a CA of $\sim 110^{\circ}$ (Fig. 6(a)). When the surface is first roughened via a chemical etch and then coated with rf-sputtered Teflon, the CA increases to $>160$ (Fig. 6(b)) due to entrapment of air in the troughs of the nanorough structure as seen in the FESEM image in Fig. 1, that reduces the area of contact with the droplet as well as the presence of low surface energy components as confirmed by XPS in Fig. 5. The contact fraction of the rfsputtered Teflon coated etched aluminum surface as calculated from Cassie-Baxter equation [21] using the contact angle data obtained was found to be $\sim 0.1$ and the water droplets just rolled off these surfaces even at the slightest tilt of the sample surface. There was no significant change in the contact angle data or the water roll-off behavior when the etched aluminum surfaces were coated with $\mathrm{ZnO}$ or $\mathrm{TiO} 2$ prior to rf-sputtered Teflon coating. These observations demonstrate the importance of the co-existence of surface roughness and low surface energy coating in obtaining superhydrophobic properties as emphasized in previous studies [8-13, 20].

Hydrophilic and superhydrophobic samples were tested for icephobicity by exposing the surfaces to simulated freezing rain in a wind tunnel. Visual analyses showed that the ice formed and continued to accumulate on the hydrophilic samples as soon as the supercooled water droplets came into contact with their surfaces.

The superhydrophobic surfaces, either with or without the oxide coatings, showed 


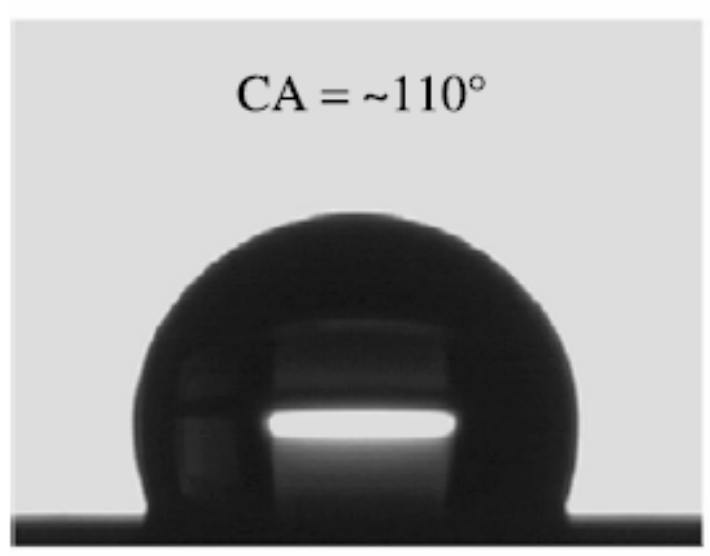

(a)

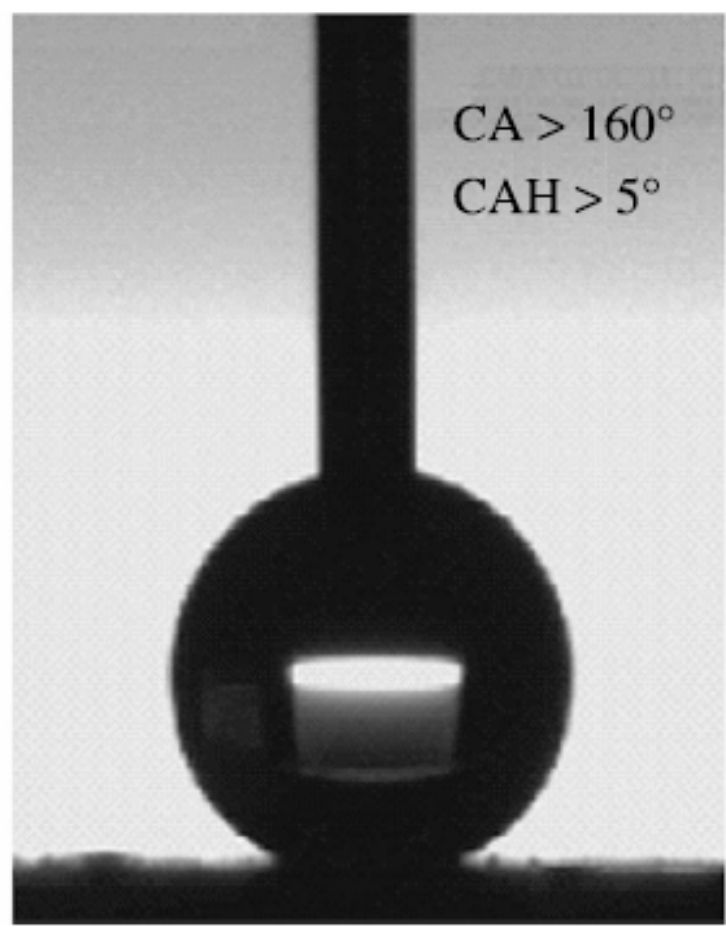

(b)

no ice formation, and, therefore, no accumulation of ice from the freezing drizzle. Petrenko and Peng [22], in their study on the adhesion of ice to hydrophilic and hydrophobic self-assembled monolayers (SAMs) on gold surfaces showed that the shear adhesion strength of ice decreased with an increase in the hydrophobicity. Study of Somlo and Gupta [23] on ice adhesion on various SAMs coated onto Al surfaces found a reduction in the tensile adhesion strength compared to the bare aluminum surfaces, emphasizing the importance of low surface energy materials, one of the two important factors in enhancing hydrophobic properties. Surface roughness, being the other important factor in enhancing the water contact angle on surfaces, has also been investigated for its role in ice adhesion [24]. However, as reported by Saito et al., an increase in surface roughness led to an increase in ice adhesion [25]. Recent studies have shown that a combination of surface roughness and a low surface energy coating leads to very high water contact angles and enhances a reduction in the adhesion strength of ice [26]. However, so far there are no reports claiming the existence of an icephobic surface on which ice would not stick at all. Figure 7 shows photographs of our superhydrophobic (with rf-sputtered Teflon coating) and hydrophilic (with no rf-sputtered Teflon coating) dielectrics coated etched aluminum surfaces (ZCEA in figure) before and after ice accumulation in the icing wind tunnel. It is clear from Fig. 7 that ice hardly forms on superhydrophobic surfaces as the quantity of ice accumulated is much smaller on the superhydrophobic surfaces than on the hydrophilic surfaces for the same period of accumulation under the same conditions. The smaller quantity of ice seen stuck at the corners may be due to surface defects or due to the untreated sample supports favoring ice formation thereby providing nucleation sites for ice growth. Since the evidence suggests that ice does not adhere to these superhydrophobic surfaces un- 

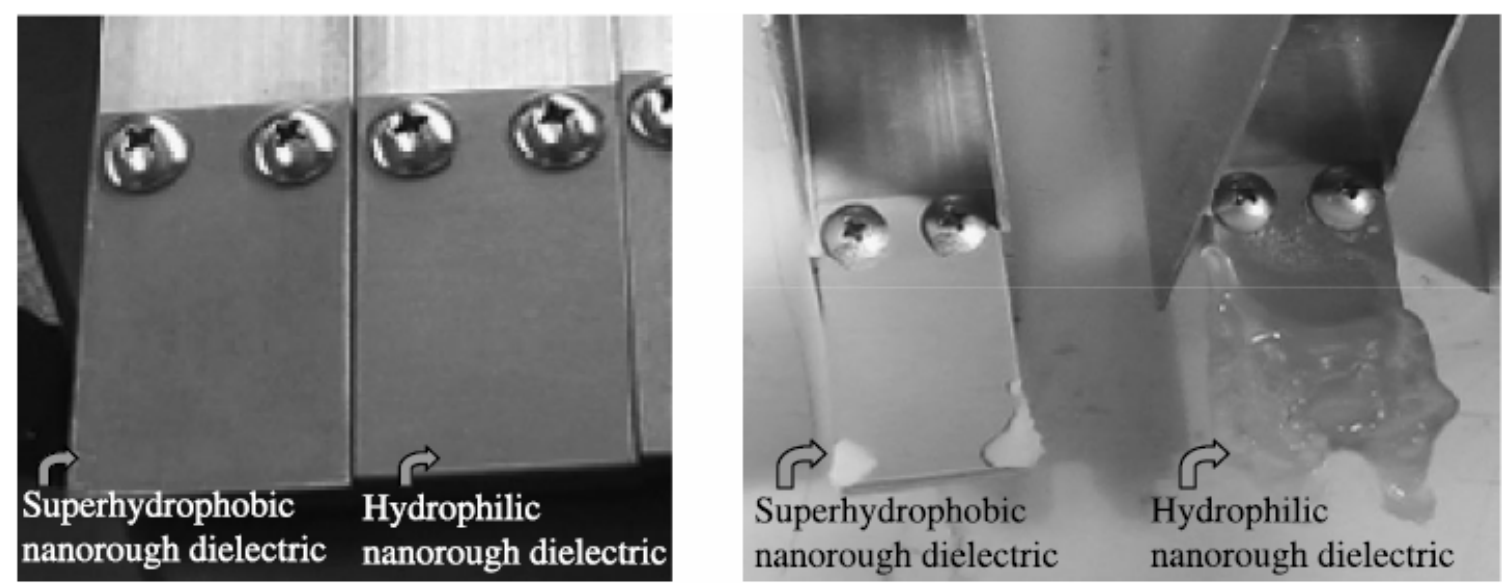

Figure 7. (Left) Sample surfaces (ZCEA in figure) before ice accumulation; (Right) superhydrophobic and hydrophilic sample surfaces after ice accumulation in the wind tunnel.

The hydrophilic samples were further tested using the CAT apparatus to evaluate the adhesion strength of ice on these surfaces. Bare aluminum was tested several times with the CAT apparatus before testing the samples to compare with literature values and the measured adhesion strength of $420 \pm 27 \mathrm{kPa}$ was found to be in good agreement with values reported in the literature [1, 19, 27]. The ice adhesion strength obtained on the nanorough Al surface was found to be $466 \pm 122 \mathrm{kPa}$.

Similarly, the ice adhesion strengths on $\mathrm{ZnO}(\varepsilon=\sim 8)$ and $\mathrm{TiO} 2(\varepsilon=\sim 80)$ coated on etched aluminum ( $\sim 82$ and $\sim 480 \mathrm{kPa}$, respectively) are similar and comparable to the value obtained on bare aluminum with the measurement errors taken into consideration (Table 2). These values obtained using CAT tests and the visual investigation in the wind tunnel show that the adhesion of ice is stronger on hydrophilic surfaces and weaker on superhydrophobic surfaces as evident from Table 2 which summarizes the ice adhesion strength values along with water contact angle and contact angle hystereses values. The '***' in Table 2 indicate that those data were unobtainable due to experimental limitations in ice adhesion tests and the impossibility to measure contact angle hysteresis on surfaces which were not superhydrophobic. Weaker adhesion strength of ice on superhydrophobic surfaces shows that on these surfaces not only the water droplets at room temperature are repelled but also the supercooled water droplets. Sarkar et al. [10] have previously shown that room temperature water droplets roll off the superhydrophobic aluminum surfaces prepared via a similar chemical etch process followed by rf-sputtered Teflon coating.

The effect of the dielectric constant on ice adhesion was also evaluated in this study by comparing the ice adhesion on etched aluminum (EA) surfaces coated with three different dielectrics TiO2 $(\varepsilon=\sim 80), \mathrm{ZnO}(\varepsilon=\sim 8)$ and Teflon $(\varepsilon=\sim 2)$. Studies indicate that materials with a lower dielectric constant will have a lower adhesion to ice due to screening of mirror charges expressed as $q^{\prime}=q \varepsilon-1 / \varepsilon+1$, where

\section{Table 2.}

Contact angle data, adhesion strength and dielectric constant values (Abbreviations: 'BA': Bare Al; 'ZCBA': ZnO-coated bare Al; 'TCBA': TiO2-coated bare Al; 'PCBA': PTFEcoated bare Al; 'EA': Etched Al; 'ZCEA': ZnO-coated etched Al; 'TCEA': TiO2-coated etched Al; 'PCEA': PTFE-coated etched Al; 'PZCEA': PTFE-coated ZCEA; and 'PTCEA': PTFE-coated TCEA) 


\begin{tabular}{lcllc}
\hline Sample & $\begin{array}{l}\text { Static water } \\
\text { contact angle }\left(^{\circ}\right)\end{array}$ & $\begin{array}{l}\text { Contact angle } \\
\text { hysteresis }\left(^{\circ}\right)\end{array}$ & $\begin{array}{l}\text { Ice adhesion } \\
\text { strength }(\mathrm{kPa})\end{array}$ & $\begin{array}{l}\text { Dielectric } \\
\text { constant }\end{array}$ \\
\hline BA & $\sim 74$ & $* * * *$ & $420 \pm 27$ & - \\
ZCBA & $\sim 31$ & $* * *$ & $450 \pm 101$ & $\sim 8$ \\
TCBA & $\sim 43$ & $* * *$ & $460 \pm 75$ & $\sim 80$ \\
PCBA & $\sim 110$ & $* * *$ & $188 \pm 12$ & $\sim 2$ \\
EA & 0 & $* * *$ & $466 \pm 122$ & - \\
ZCEA & 0 & $* * *$ & $482 \pm 125$ & $\sim 8$ \\
TCEA & 25 & $* * *$ & $480 \pm 60$ & $\sim 80$ \\
PCEA, PZCEA, & $>160$ & $<5$ & $* * *$ & $\sim 2$ \\
\multicolumn{1}{l}{ PTCEA } & & & & \\
\hline
\end{tabular}

**** These data were unobtainable.

$q^{\prime}$ is the image charge produced on the dielectric surface by a charge $q$ on the ice surface and $\varepsilon$ is the dielectric constant of the insulator [3]. TiO2-coated etched aluminum surfaces (TCEA) $(\varepsilon=\sim 80)$ showed a very high ice adhesion strength of $480 \pm 60 \mathrm{kPa}$. When the etched aluminum was coated with the lower dielectric constant material $\mathrm{ZnO}$ $(\varepsilon=\sim 8)(Z C E A)$, the adhesion strength $(482 \pm 125 \mathrm{kPa})$ remained high. However, when the etched aluminum was coated with rf-sputtered Teflon (PCEA), with the lowest dielectric constant $(\varepsilon=\sim 2)$, the ice adhesion as discussed above was found to be almost zero. Similar behavior was found when the dielectrics-coated etched aluminum surfaces were further coated with rf-sputtered Teflon.

In order to eliminate the effect of roughness on the surfaces tested, we performed the same experiments on flat (bare) aluminum surfaces coated with $\mathrm{ZnO}$, or $\mathrm{TiO} 2$ or rfsputtered Teflon. From Table 2, it can be seen that flat ZnO (ZCBA) and TiO2 (TCBA) surfaces showed a high and comparable adhesion strength of $450 \pm 101 \mathrm{kPa}$ and $460 \pm$ $75 \mathrm{kPa}$, respectively, both comparable to $420 \pm 27 \mathrm{kPa}$ obtained on bare Al. Therefore, varying $\varepsilon$ from $\sim 80$ ( $\mathrm{TiO} 2)$ to $\sim 8(\mathrm{ZnO})$ showed no effect on ice adhesion, however, the adhesion strength is considerably lowered $(\sim 188 \mathrm{kPa})$ when bare Al surfaces were coated with rf-sputtered Teflon $\AA$, a dielectric itself with a dielectric constant as low as $\varepsilon=\sim 2$. Therefore, the dielectric constant of a material is found to have an influence on ice adhesion; however, a change in the dielectric constant from 80 to 8 does not have a significant effect, although a reduction in the dielectric constant to 2 does. The experimental observation is complemented by the theoretical prediction in the literature [3] based on mirror charges. The mirror charges $q$ ' on $\mathrm{ZnO}(\varepsilon=\sim 8)$ and TiO2 $(\varepsilon=\sim 80)$, are found to be $0.78 q$ and $0.98 q$, respectively based on theoretical predictions. However, for a dielectric with $\varepsilon=\sim 2$ (Teflon), the image charge $q$ ' is predicted to be only $0.33 q$, which is one-third of the charge on ice as compared to the other two dielectrics studied for which $q$ ' is nearly the same as $q$.

Another interesting observation in this study is that an etched aluminum surface becomes superhydrophobic once a coating of Teflon is applied over it, irrespective of the presence or absence of an intermediate layer of $\mathrm{TiO} 2$ or $\mathrm{ZnO}$. Teflon has a very low surface energy of $\sim 18 \mathrm{~mJ} / \mathrm{m} 2[7,21]$ and, therefore, has very low affinity towards water. The interaction depth of water with low surface energy components as reported by Bain and Whitesides is only $\sim 0.5 \mathrm{~nm}$ [28]. Therefore, water and hence ice does not 'sense' the substrate material underlying the Teflon layer, which nearly completely masks the substrate in the XPS spectra, indicating a thickness of only a few $\mathrm{nm}$. 


\section{Conclusions}

Aluminum surfaces (with or without a $\mathrm{TiO} 2$ or $\mathrm{ZnO}$ coating) were rendered superhydrophobic by creating a certain nanoroughness on the aluminum surface by chemical etching and by lowering the surface energy by applying a coating of rfsputtered Teflon. The creation of a rough nanostructure was confirmed from FESEM images and the presence of the coating of the oxides as well as rf-sputtered Teflon was confirmed by XPS analyses. Contact angles $>160 \circ$ and contact angle hystereses $<5 \circ$ were obtained on these surfaces which demonstrated the 'lotus effect'.

Icephobicity tests conducted on these surfaces showed no formation of ice on superhydrophobic surfaces, indicating zero adhesion. However, as expected, ice formed and accumulated on hydrophilic samples demonstrating very high adhesion strengths of $>400 \mathrm{kPa}$, values similar to that obtained on bare aluminum. Therefore, superhydrophobic surfaces, governed by geometry and chemistry, have shown to be promising candidates as icephobic surfaces. The study of the effect of the dielectric constant on ice adhesion showed that the dielectric constant did have an effect but it was apparent only when the dielectric constant was reduced to the very low value of 2 for Teflon.

\section{Acknowledgements}

The authors thank the Natural Sciences and Engineering Research Council of Canada (NSERC), Hydro-Québec, University of Québec at Chicoutimi (UQAC) and all the partners of Industrial Chair on Atmospheric Icing of Power Network Equipment (CIGELE) and Canada Research Chair on Engineering of Power Network Atmospheric Icing (INGIVRE) for their financial support.

\section{References}

1. M. Javan-Mashmool, C. Volat and M. Farzaneh, Hydrol. Process. 20, 645 (2006).

2. Z. Peter, PhD Dissertation in Engineering, University of Quebec at Chicoutimi (UQAC) (2006).

3. I. A. Ryzhkin and V. F. Petrenko, J. Phys. Chem. B 101, 6267 (1997).

4. D. Quéré, Rep. Prog. Phys. 68, 2495 (2005).

5. Y. Zheng, X. Gao and L. Jiang, Soft Matter 3, 178 (2007).

6. X. Gao and L. Jiang, Nature 432, 36 (2004).

7. W. Barthlott and C. Neinhuis, Planta 202, 1 (1997).

8. N. Saleema and M. Farzaneh, Appl. Surf. Sci. 254, 2690 (2008).

9. A. Safaee, D. K. Sarkar and M. Farzaneh, Appl. Surf. Sci. 254, 2493 (2008).

10. D. K. Sarkar, M. Farzaneh and R. W. Paynter, Mater. Lett. 62, 1226 (2008).

11. D. K. Sarkar and R. W. Paynter, J. Adhesion Sci. Technol. 24, 1181 (2010).

12. P. F. Rios, H. Dodiuk, S. Kenig, S. McCarthy and A. Dotan, J. Adhesion Sci.

Technol. 20, 563

(2006).

13. P. F. Rios, H. Dodiuk, S. Kenig, S. McCarthy and A. Dotan, J. Adhesion Sci.

Technol. 21, 399

(2007).

14. A. Carré and K. L. Mittal (Eds), Superhydrophobic Surfaces. VSP/Brill, Leiden (2009).

15. T. P. Alexander, T. J. Bukowski, G. Teowee, D. R. Uhlmann, K. C. McCarthy, J. Dawley and

B. J. J. Zelinski, Proc. IEEE IntI Symp. on Applns. of Ferroelect. 2, 585 (1996).

16. D. K. Sarkar, D. Brassard, M. A. El-Khakani and L. Ouellet, Thin Solid Films 515, 4788 (2007). 
17. M. A. El-Khakani, D. K. Sarkar, L. Ouellet and D. Brassard, US Patent 7, 101, 754 (2006).

18. "User Manual for Drop Shape Analyzer" Krüss GmbH, DSA1 v1.9-03 (2004).

19. C. Laforte and A. Beisswenger, in: Proceedings of the International Workshop on Atmospheric

Icing of Structures (IWAIS XI), Montréal, Canada, p. 357 (2005).

20. X.-M. Li, D. Reinhoudt and M. Crego-Calama, Chem. Soc. Rev. 36, 1350 (2007).

21. A. B. D. Cassie and S. Baxter, Trans. Faraday Soc. 40, 546 (1944).

22. V. Petrenko and S. Peng, Can. J. Phys. 81, 387 (2003).

23. B. Somlo and V. Gupta, Mech. Mater. 33, 471 (2001).

24. C. Laforte, J.-L. Laforte and J.-C. Carrier, in: Proceedings of the International

Workshop on Atmospheric

Icing of Structures (IWAIS X), Brno, Czech Republic, p. 6 (2002).

25. H. Saito, K. Takai and G. Yamauchi, Surf. Coat. Int. 80 (4), 168 (1997).

26. D. K. Sarkar and M. Farzaneh, J. Adhesion Sci. Technol. 23, 1215 (2009).

27. H. H. G. Jellinek, J. Colloid Sci. 14, 268 (1959).

28. C. D. Bain and G. M. Whitesides, J. Am. Chem. Soc. 110, 5897 (1988). 